\title{
Genetics of rheumatoid arthritis: 2018 status
}

\author{
Yukinori Okada, ${ }^{1,2}$ Stephen Eyre, ${ }^{3}$ Akari Suzuki, ${ }^{2}$ Yuta Kochi, ${ }^{2}$ Kazuhiko Yamamoto ${ }^{2}$
}

\section{Handling editor Josef S \\ Smolen}

'Department of Statistical Genetics, Osaka University Graduate School of Medicine, Osaka, Japan

${ }^{2}$ Laboratory for Autoimmune Diseases, RIKEN Center for Integrative Medical Sciences, Yokohama, Japan

${ }^{3}$ Division of Musculoskeletal and Dermatological Sciences, School of Biological Sciences, The University of Manchester, Manchester, UK

\section{Correspondence to}

Dr Kazuhiko Yamamoto, Laboratory for Autoimmune Diseases, RIKEN Center for Integrative Medical Sciences, Yokohama 230-0045, Japan; kazuhiko.yamamoto@riken.jp

Received 3 September 2018 Revised 6 November 2018

Accepted 7 November 2018 Published Online First

8 December 2018

\section{ABSTRACT}

Study of the genetics of rheumatoid arthritis (RA) began about four decades ago with the discovery of HLA-DRB1. Since the beginning of this century, a number of nonHLA risk loci have been identified through genome-wide association studies (GWAS). We now know that over 100 loci are associated with RA risk. Because genetic information implies a clear causal relationship to the disease, research into the pathogenesis of RA should be promoted. However, only $20 \%$ of GWAS loci contain coding variants, with the remaining variants occurring in non-coding regions, and therefore, the majority of causal genes and causal variants remain to be identified. The use of epigenetic studies, high-resolution mapping of open chromatin, chromosomal conformation technologies and other approaches could identify many of the missing links between genetic risk variants and causal genetic components, thus expanding our understanding of RA genetics.

\section{INTRODUCTION}

Rheumatoid arthritis (RA) is an inflammatory rheumatic disease that causes chronic synovial inflammation, eventually leading to disabling joint destruction as well as systemic complications. ${ }^{1}$ Most epidemiologic studies indicate that the prevalence of RA is $0.5 \%-1.0 \%$. Between $70 \%$ and $80 \%$ of patients with RA have autoantibodies such as rheumatoid factor and anti-citrullinated protein antibodies (ACPA), suggesting that RA is an autoim-

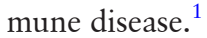

The majority of rheumatic diseases involve complex traits in which multiple genetic and environmental factors interact. Twin studies have estimated that the heritability of RA is $\sim 60 \% .^{2}$ This applies primarily to patients with RA who are positive for ACPAs, whereas the heritability of seronegative RA appears to be lower. Since 2007, rapid advances in genome-wide association study (GWAS) technologies have facilitated the identification of hundreds of genetic risk factors for many complex diseases. ${ }^{3}$ To date, more than 100 genetic loci have been associated with RA. ${ }^{4}$ However, the relationship of these loci to the disease remains to be elucidated.

As a genetic factor has a clear causal relationship to RA, it is important to understand the pathologic process from a genomic standpoint. Recent studies of complex trait diseases have indicated that many disease susceptibility variants regulate the expression levels of a number of genes that function in a cell-specific manner. ${ }^{5}$ Furthermore, the epigenome is thought to play an important role in this phenomenon. Obtaining a more thorough understanding of this complex regulatory network is vital to determining which genes and cell types play pivotal roles in RA, thus helping to identify key pathways that drive RA and enable stratification of patients into groups based on the causative pathways. Here, we describe the state of genetic research to date, envisaging a better understanding of the pathogenesis of RA.

\section{THE CURRENT STATUS OF RA GENETICS}

Studies investigating the correlation between variations in human genome sequences and RA casecontrol phenotypes have identified a number of genetic variants associated with RA susceptibility. Here, we briefly review the history of RA genetics research (table 1). The RA risk locus was first identified in around 1980, and this research elucidated the role of HLA-DRB1 alleles in the major histocompatibility (MHC) locus. ${ }^{6}$ In the early 2000 s, the International HapMap Project consolidated the map of human genome sequence variations in multiple populations, ${ }^{7}$ which enabled the unbiased genome-wide screening of genetic variants (mostly represented as single nucleotide polymorphisms (SNP)) associated with human phenotypes. ${ }^{8}$ In a visionary early GWAS of RA, ${ }^{9} 10$ PADI4 was identified as an initial non-MHC RA risk locus in the Japanese population. ${ }^{9}$ Then, large-scale GWAS using commercial microarrays were conducted for a wide range of human complex traits including autoimmune diseases and RA. ${ }^{11-13}$

Table 1 Overview in history of RA genetics discovery

\begin{tabular}{llllll}
\hline Publication year & Trait & Ethnicity & Approach & Representative findings in RA genetics & Reference \\
\hline Around 1980 & Single & Single & Single locus & Identification of HLA-DRB1 & 6 \\
2003 & Single & Single & Early GWAS & Identification of PADI4 and FCRL3 & 910 \\
2007 & Single & Single & GWAS with SNP array & Identification of TNFAIP3, TRAF1-C5 and STAT4 & $11-13$ \\
2010 & Single & Transethnic & GWAS meta-analysis & Works by RACl, GARNET and immunochip consortia & $18-20$ \\
2011 & Multiple & Single & GWAS meta-analysis & Shared risk loci between RA and coeliac disease & 32 \\
2014 & Single & Transethnic & GWAS meta-analysis & Identification of >100 RA risk loci & 4 \\
2015 & Multiple & Single & Polygenic analysis & Negative genetic correlation between RA and SCZ & 3637 \\
Future & Multiple & Transethnic & Polygenic analysis & - & - \\
\hline GARNET, Genetics and Allied research in Rheumatic diseases Networking consortia; GWAS, genome-wide association study; RA, rheumatoid \\
arthritis; RACl, the Rheumatoid Arthritis Consortium International; SCZ, schizophrenia; SNP, single nucleotide polymorphism.
\end{tabular}


While early RA GWASs were conducted separately for each cohort, association signals in the RA risk loci were largely replicated among the multiple cohorts, ${ }^{14}{ }^{15}$ suggesting that meta-analyses of multiple GWASs would increase the statistical power. By applying in silico imputation of SNPs not genotyped in the GWAS data with an independent reference panel with high-density SNPs, ${ }^{716}$ genotype data for a unified set of millions of genome-wide SNPs can be obtained, which were used for the GWAS meta-analysis. ${ }^{17}$ Since 2010 , several collaborative efforts have been initiated with the goal of organising data from multiple RA GWASs and meta-analyses of these data identified a number of RA risk genes. ${ }^{18-20}$ These initiatives also contributed to the construction of a reliable network within the community of RA genetics researchers.

The accumulation of RA GWAS meta-analysis results in each of these populations provided evidence of a shared genetic background among patients with RA in different populations. The researchers found multiple genetic loci that confer significant RA risk in multiple ancestry (eg, reidentification of PADI4 as an RA risk locus in Europeans) ${ }^{20}$ and its interaction with population-specific environmental factors. ${ }^{21}$ Further, numerous genome-wide SNPs weakly but significantly shared disease risk between the populations. ${ }^{19} 22$ It was also suggested that single-population GWAS were mostly underpowered, and transethnic data integration was warranted to gain statistical power to identify the trait-associated loci and to unveil hidden disease aetiology. These observations provided strong motivation for a transethnic study to integrate multiple populations. ${ }^{18-25}$ Thus, we conducted an initial transethnic RA GWAS meta-analysis involving >100 000 subjects from European and Asian populations. ${ }^{4}$ This study identified $>100$ RA risk genetic loci, demonstrating the value of human transethnic GWAS. Figure 1 shows a list of presumed RA risk genes identified to date, grouped according to chromosomal position. This plot helped us visually grasp the 'landscape' of RA genetics; that is, the studies in European and Asian populations both substantially contributed to the identification of RA risk genes. However, we must note that more recent transethnic studies have focused only on Europeans and Asians (mostly from East Asia), and coverage of worldwide populations remains limited. Nevertheless, the current transethnic GWAS are still underpowered to dissect overall genetic architecture of human complex traits. ${ }^{26}$ Future efforts should therefore integrate other ethnicities. $^{27-30}$

Another approach to expand understanding of RA genetics is the use of cross-trait analyses to identify genetic correlations with other human complex traits. It has been reported that genetic variants associated with RA likely also confer risk to other diseases (ie, pleiotropy). ${ }^{31}$ This includes autoimmune diseases, and allergic diseases, biomarkers (eg, neutrophil count and C-reactive protein) and cancers. ${ }^{4}$ An interesting approach for a GWAS meta-analysis examining RA and coeliac disease identified a number of loci with pleiotropic effects. ${ }^{32}$ A recently developed method, polygenic analysis, enables evaluation of the top-associated variants and numerous genome-wide SNPs with relatively small effect sizes. ${ }^{33}$ Specifically, the introduction of the polygenic risk score (PRS) ${ }^{34}$ and linkage disequilibrium score regression (LDSC) ${ }^{35}$ methods enabled quantification of genetic correlations among different phenotypes. PRS calculates a disease risk score for each subject included in the GWAS by integrating the genotype with the corresponding susceptibility risks of genome-wide SNPs. The LDSC method does not assess an individual's genotype and instead uses summary statistics of genome-wide SNPs in the GWAS results (ie, ORs and p values) and estimates shared genetic backgrounds between phenotypes and their cell-type specificity. Application of the PRS and LDSC methods pointed to a negative genetic correlation between RA and schizophrenia, ${ }^{3637}$ which could partly explain the relatively lower comorbidity between these two diseases previously highlighted by epidemiologic studies. ${ }^{38}$

\section{MHC COMPLEX: GENETICS AND BIOLOGY}

The MHC region at chromosome 6 confers a distinctive and strong genetic risk when compared with other RA risk loci, explaining $30 \%-50 \%$ of total genetic risk of RA. ${ }^{39}$ Within the MHC, a class II classical human leucocyte antigen (HLA) gene, HLA-DRB1, explains the majority of RA risk. Which combinations of HLA-DRB1 variants could best explain disease risk has been a long-standing subject of debate. In 1987, the shared epitope (SE) hypothesis was introduced to show the risk associated with the specific amino acid sequence at positions 70-74 of HLA-DR $\beta 1 .^{6}$ Although the SE hypothesis was supported by data from populations around the world, there was controversy regarding the RA risk associated with non-SE HLA alleles (eg, HLA-DRB1*09:01 in Asians). ${ }^{40}$ GWAS have identified that ACPA-positive and ACPA-negative RA showed dramatic difference in genetic backgrounds as analogous to heterogeneity in clinical manifestations, of which association signals were most apparent at the MHC region. ${ }^{41}$

The recent development of an HLA imputation method led to fine-mapping of genetic risks within the MHC for a variety of immune-related diseases. ${ }^{42}{ }^{43}$ Similar to SNP genotype imputation, the HLA imputation method computationally imputes risk to unobserved HLA gene variants according to neighbouring GWAS SNP genotypes in the MHC and an imputation reference panel (figure 2A). By applying HLA imputation, one can assess both alleles and amino acid polymorphisms of all HLA genes included in the reference panel for all samples with available GWAS data. ${ }^{43}$

Application of HLA imputation to large-scale RA GWAS data produced several interesting findings. (1) Most of the risk of ACPA-positive RA could be explained by amino acid polymorphisms at positions 11 and 13 of HLA-DR $\beta 1$, rather than at the well-known positions 71 and 74 as implicated by the SE hypothesis (figure $2 \mathrm{~B}$ ). ${ }^{44}$ Residues at HLA-DR $\beta 1$ positions 11 and 13 tag several SE alleles such as HLA-DRB $1 * 01$ and $* 04,{ }^{45}$ implying that the amino acid model could be interpreted as extension of the SE hypothesis. (2) Although the MHC-associated genetic risks of ACPA-positive and ACPA-negative RA were found to be heterogeneous, they could be explained by the same HLA-DR $\beta 1$ amino acid positions but different risk-conferring residues. ${ }^{46}$ This may suggest other autoimmune responses other than ACPA contributes to ACPA-negative RA. (3) Risk HLA variants were found to be shared among populations more than expected (eg, Europeans, Asians and African Americans), which closed the debate regarding risk and ethnic heterogeneity in HLA alleles. $^{45} 47$ (4) In addition to HLA-DRB1, amino acid polymorphisms in other classical HLA genes, such as HLA-DPB1, HLA-B and HLA-A, confer risk for ACPA-positive RA. ${ }^{44-46}$ (5) Finally, a coding variant in a non-classical HLA gene (HLA-DOA) that alters the gene's expression level also confers risk for ACPA-positive RA (figure 2C). ${ }^{48}$

Next-generation sequencing (NGS) technology represents a promising tool for use in future MHC fine-mapping studies. ${ }^{49}$ Current imputation reference panels include limited numbers of classical HLA genes. However, the MHC includes a number of HLA-related genes, including non-classical HLA genes (eg, $H L A-E / F / G)$, HLA-like genes (eg, MICA) and pseudo-HLA genes 

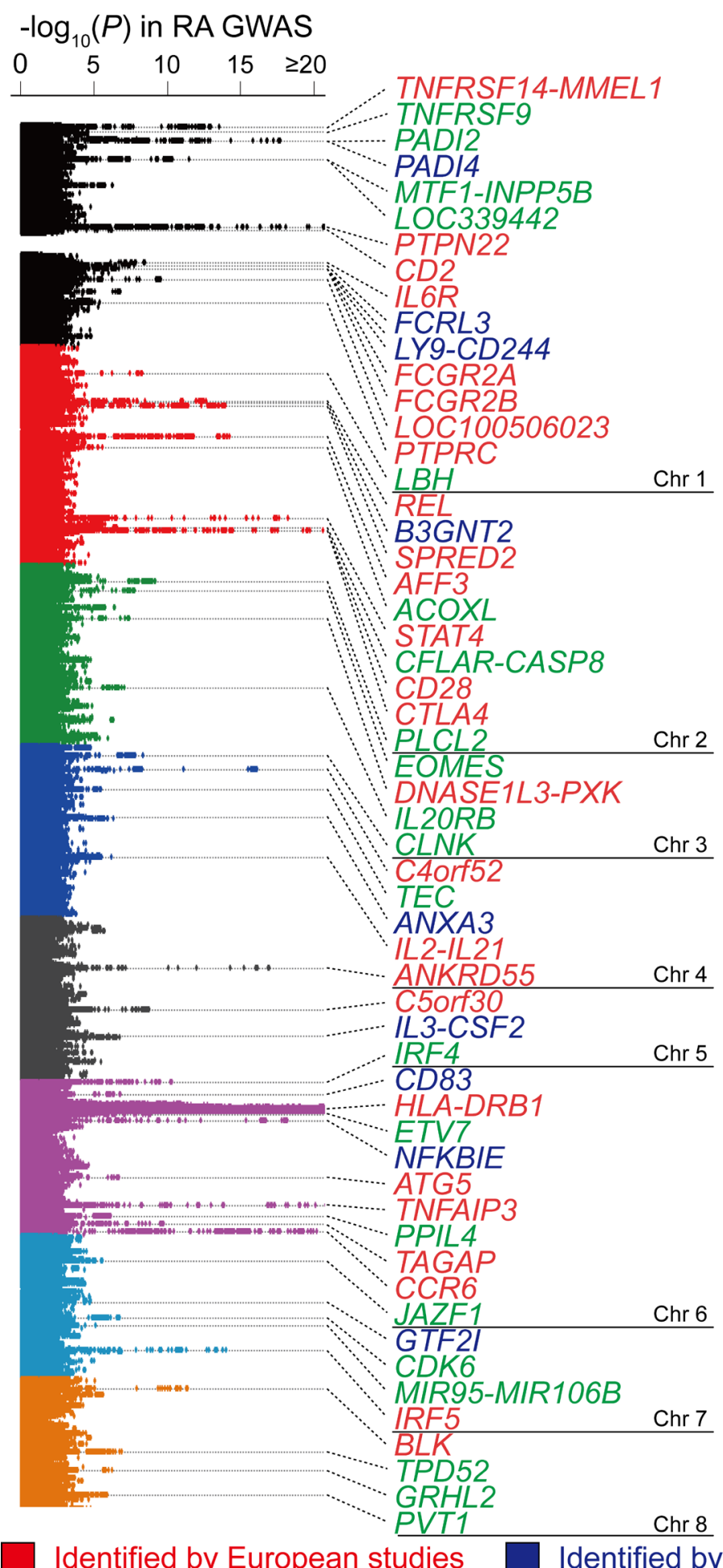

$-\log _{10}(P)$ in RA GWAS
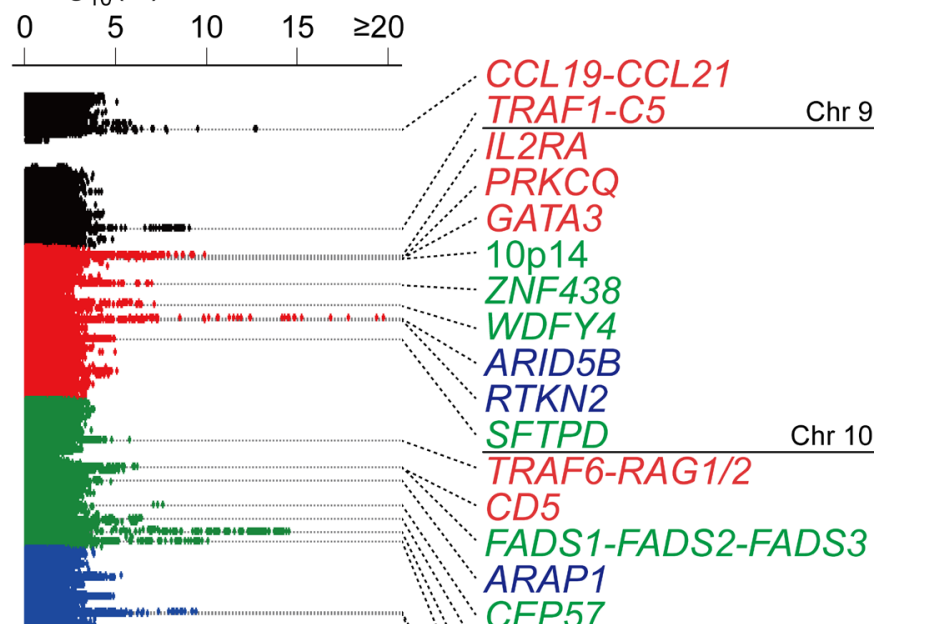

CEP57

ATM

CXCR5

ETS1

CDK2

CDK4

SH2B3-PTPN11 Chr 12

\begin{tabular}{ll}
\hline COG6 & Chr 13 \\
\hline
\end{tabular}

PRKCH

RAD51B

PLD4-AHNAK2 Chr 14

RASGRP1

LOC145837 Chr 15

TXNDC11

- MIR762

IRF8

C1QBP

$M E D 1$

\begin{tabular}{ll} 
IKZF3-CSF3 Chr 17 \\
\hline
\end{tabular}

PTPN2

\begin{tabular}{ll} 
CD226 & Chr 18 \\
\hline
\end{tabular}

TYK2

\begin{tabular}{ll} 
ILF3 & Chr 19 \\
\hline
\end{tabular}

\begin{tabular}{ll}
$C D 40$ & $\mathrm{Chr} 20$ \\
\hline
\end{tabular}

IFNGR2

RCAN1

RUNX1

UBASH3A

ICOSLG-AIRE Chr 21

UBE2L3-MIR301B

IL $2 R B$

SYNGR1

P2RY10

IRAK1

Chr 22

Identified by European studies $\square$ Identified by Asian studies $\square$ Identified by trans-ethnic studies

Figure 1 A current catalogue of rheumatoid arthritis (RA) risk gene loci. A list of RA risk 106 gene loci identified to date along with a Manhattan plot of the transethnic genome-wide association study (GWAS) meta-analysis of RA. ${ }^{48-2022-25}$ The significance of each single nucleotide polymorphism (SNP) in the RA GWAS is indicated on a logarithmic scale (X-axes). RA risk genes initially identified in the European, Asian and transethnic studies are coloured red, blue and green, respectively.

(eg, HLA-DRB6), as well as key immunity-related genes (eg, TNF and $C 4 A-C 4 B)$. NGS-based approaches could identify variants with higher resolution, thus warranting their incorporation in reference panels.

\section{WHAT CAN WE LEARN FROM GENETICS OF RA?}

Missense variants, which alter the amino acid sequence of a coding gene, are common functional variants that can be pathogenic. The most important and well-characterised missense risk variant in RA may be PTPN22 R620W $(1858 \mathrm{C} \rightarrow \mathrm{T})$ although this risk variant is extremely rare in east Asian populations. ${ }^{50}$ PTPN22 encodes a protein tyrosine phosphatase that is expressed in haematopoietic cells and acts as a negative regulator of antigen receptor signalling in $\mathrm{T}$ and $\mathrm{B}$ cells. ${ }^{51}$ The risk allele $620 \mathrm{~W}$ is a gain-of-function variant, as both TCR and BCR signalling are reduced in cells of risk allele carriers. ${ }^{52} 53$ This 


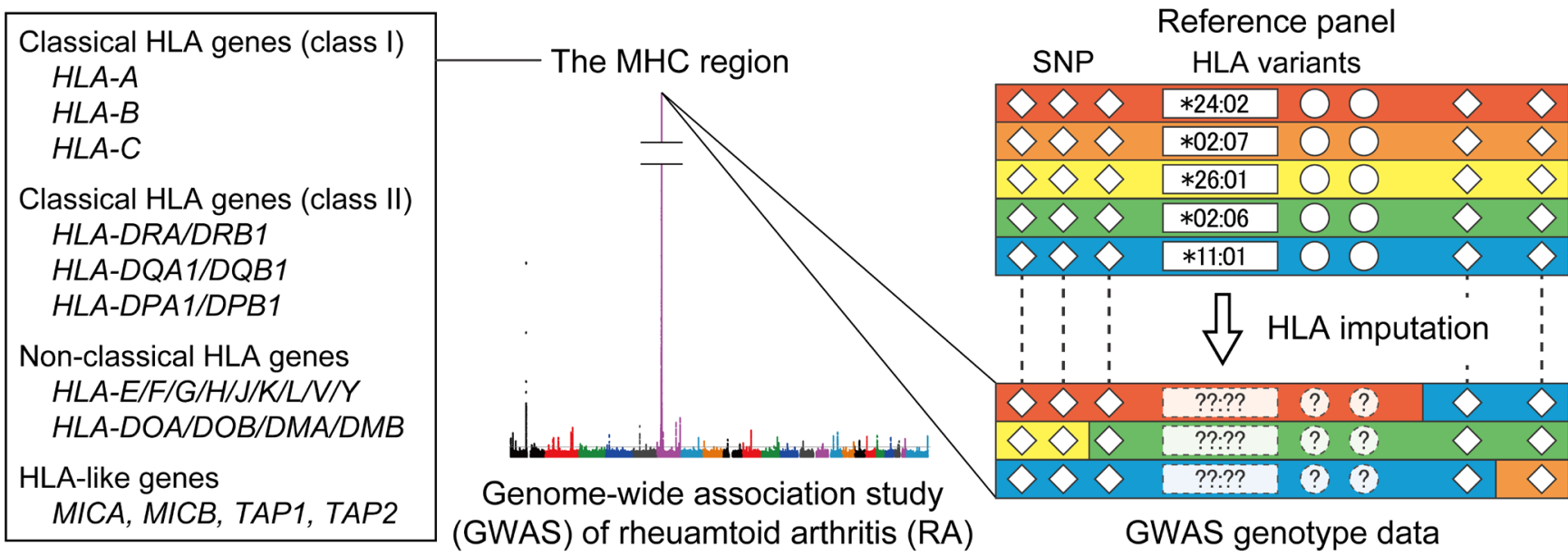

B

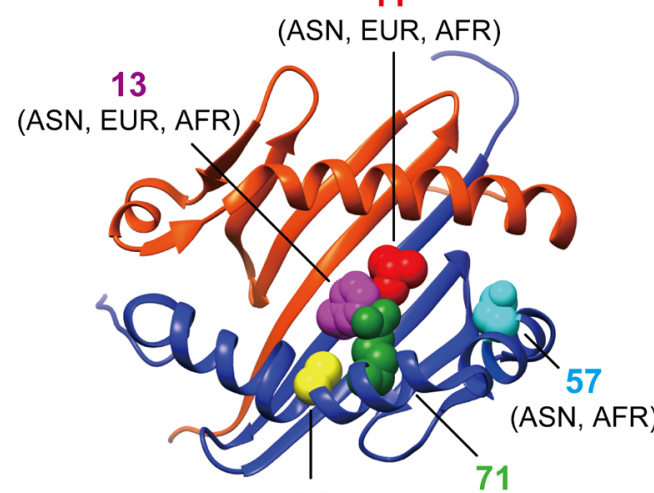

(ASN, EUR)

(EUR)

\section{HLA-DRß1 amino acid positions with risk of ACPA-positive RA}

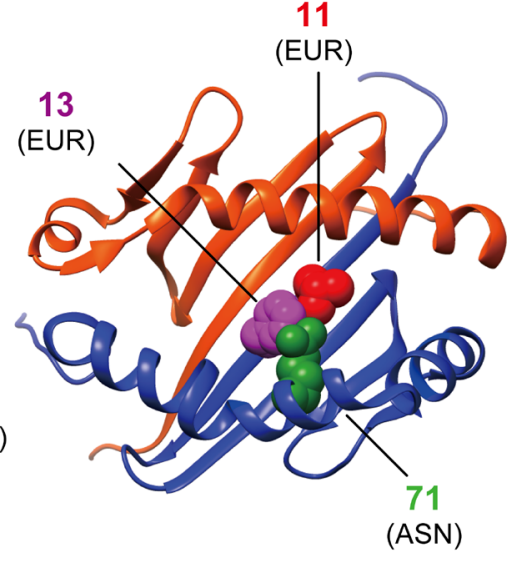

HLA-DR 1 amino acid positions
with risk of $A C P A-n e g a t i v e ~ R A$
C
Amino acid change in the classical HLA genes

HLA-DRB1, HLA-DPB1 $H L A-B, H L A-A$<smiles>[3H]</smiles>

Dosage change in the non-classical HLA gene

HLA-DOA<smiles>C1=CC=C1</smiles>

\section{Onset of ACPA-positive RA}

Figure 2 Rheumatoid arthritis (RA) genetic risk in the major histocompatibility (MHC) region revealed using the human leucocyte antigen (HLA) imputation method. Illustration of the roles of the MHC complex region and human leucocyte antigen (HLA) genes in RA genetics. (A) The MHC region at 6 p23 harbours numerous immune-related genes, including HLA genes. One can computationally estimate genotypes of the HLA variants using the HLA imputation method without any additional cost other than that associated with single nucleotide polymorphism (SNP) microarray typing. (B) Amino acid polymorphisms at specific positions in the classical HLA genes confer risk of RA (eg, positions 11 and13 at HLA-DRß1), which are generally shared among multiple populations (Asians (ASN), Europeans (EUR) and Africans (AFR)). It is interesting that different residues at the same amino acid positions confer differential risk of anti-citrullinated protein antibody (ACPA)-positive and negative RA. (C) Dosage change in the nonclassical HLA gene also confers risk of ACPA-positive RA.

attenuation in antigen receptor signalling affects the clonal selection of lymphocytes and the appearance of autoreactive cells. ${ }^{54}$ Reduced TCR signalling resulting in impaired regulatory function has also been observed in regulatory T cells. ${ }^{55}$ Moreover, the PTPN22 variant has also been associated with reduced TLR7 signalling in plasmacytoid dendritic cells ${ }^{56}$ as well as hypercitrullination in peripheral blood mononuclear cells. ${ }^{57}$ Thus, the variant's effect essentially depends on cellular context, and these compound effects in multiple cell types may contribute at each step of pathogenesis. Surprisingly, knock-in mice with the corresponding allele (Ptpn22 619W) exhibit enhanced antigen receptor signalling, ${ }^{58}$ perhaps due to enhanced degradation of the Ptpn22 619W product. ${ }^{59}$ The phenotype is contrary to that of human lymphocytes but similar to that of lymphocytes in Ptpn22-deficient mice, indicating that Ptpn22 619W is a loss-offunction variant in mice.

Although functional analysis of missense variants is straightforward, only $\sim 20 \%$ of GWAS RA loci encompass coding variants. ${ }^{4}$ In the majority of GWAS loci, as described above, disease-causing variants regulate gene expression. Indeed, $\sim 50 \%$ of RA risk SNPs colocalise with expression quantitative trait loci (eQTL) found in peripheral blood mononuclear cells. ${ }^{4}$ eQTL are defined as genetic variants that alter the expression levels or splicing patterns of a specific gene. Missense variants in the risk loci are sometimes not causal but simply tag the true causal variants having eQTL effects. Therefore, it is rational to integrate data from GWAS and eQTL studies to elucidate the disease mechanism. This approach enables connection of a GWAS variant to a responsible gene and identification of the responsible cell types, as observed eQTL effects are sometimes cell-type specific (figure 3A). Furthermore, eQTL variants can also indicate the direction of gene regulation (figure $3 \mathrm{~B}$ ). For example, upregulation of eQTL risk genes such as STAT4 ${ }^{60}$ and $C C R 6^{61}$ has been linked to upregulated production of inflammatory cytokines in patients. As individual effects of eQTL variants are small, examining the overall effects of eQTL variants in the disease was attempted by combining the whole data sets from a GWAS and an eQTL study. ${ }^{62}$ This analysis evaluated the effects 
A

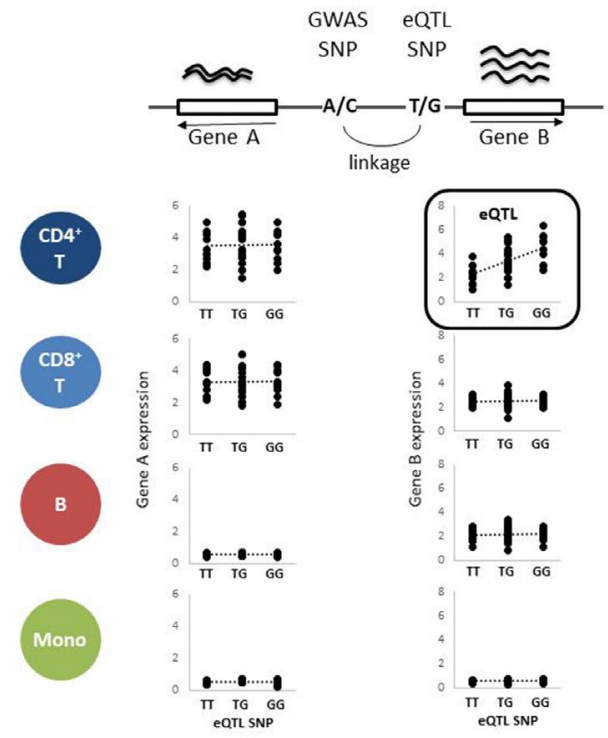

B

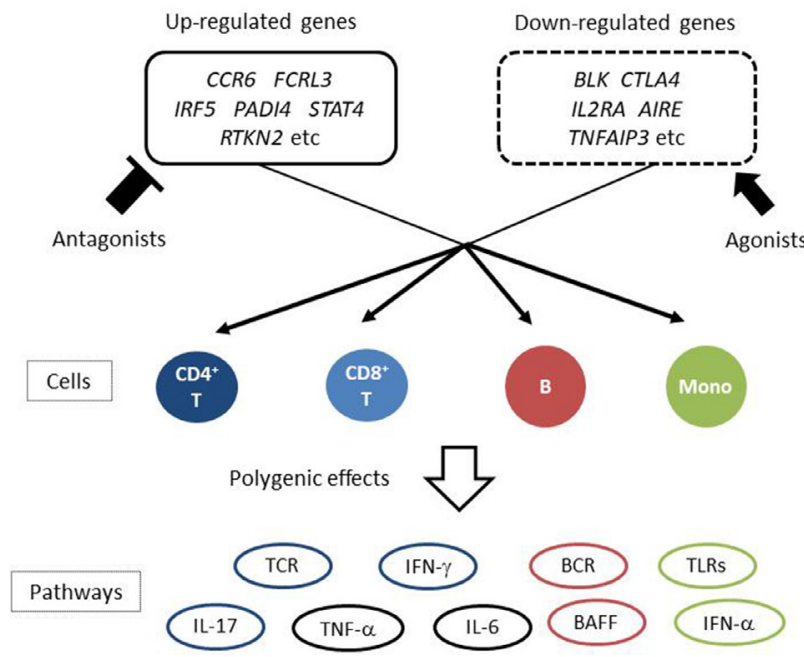

Figure 3 Integration of GWAS and eQTL data enhances understanding of the disease mechanism. (A) Identification of diseaseassociated genes and cells in the GWAS loci using eQTL data. In this case, the eQTL SNP, which is in linkage disequilibrium with the GWAS SNP, has an eQTL effect for gene $B$ (the responsible gene) in CD4+T cells (the responsible cells). (B) Polygenic effects of disease-associated eQTL genes on disease-related cells and pathways. Upregulated genes are eQTL genes whose expression is upregulated in the risk allele and could be targeted by an antagonist (vice versa for downregulated genes). eQTL, expression quantitative trait loci; GWAS, genome-wide association studies; IFN, interferon, IL, interleukin; SNP, single nucleotide polymorphism; TNF, tumour necrosis factor.

of multiple variants on inflammatory cytokine pathways in each lymphocyte subset and demonstrated that polygenic effects of eQTL genes upregulate the tumour necrosis factor (TNF)- $\alpha$ pathway in CD4 + T cells.

While researchers believe that disease risk variants are responsible for the heterogeneous aetiology of RA, the use of genetic data in the prediction of clinical phenotype is challenging. Previous GWAS examining the response to biologics (mainly anti-TNF therapy) provided unsatisfactory evidence, ${ }^{63-67}$ which may suggest that genetic background of RA onset and that of clinical response are distinct. A crowdsourced collaborative assessment of SNP data to predict
anti-TNF treatment response was performed. ${ }^{68}$ However, no significant genetic contribution towards prediction accuracy has been obtained.

\section{FURTHER GENETIC ASSIGNMENT OF RA SUSCEPTIBILITY}

A surprising GWAS finding is that approximately $80 \%$ of RA risk variants occur in non-coding regions (figure 4A). ${ }^{4}$ This physical location effect, coupled with data demonstrating that associated SNPs are actually highly correlated with an often large number of other variants, means that GWAS have not conclusively identified a causal gene or causal variant.

Studies are now well under way to provide this missing link to SNP, gene and mechanism. ${ }^{69}$ As discussed, eQTL studies can correlate a specific associated allele with gene expression or isoform splicing pattern, linking GWAS variants to causal genes with implication in functional aetiology, in particular cell types and stimulatory conditions. In addition, epigenetics and molecular biology are now routinely employed to annotate putative GWAS causal SNPs to active DNA and genes. Resources such as Encyclopedia of DNA Elements (ENCODE) ${ }^{70}$ Roadmap $^{71}$ and Blueprint $^{72}$ use techniques such as DNaseHS and ATAC-seq to map open active DNA in a range of cell types. These technologies distinguish active, open chromatin from the active form due to the fact that open chromatin is more readily cut (DNaseHS) or more readily accessible (ATAC-seq), creating sequencing libraries enriched for open DNA. Histone modifications, markers for gene regulators, can be mapped in different cell types using chromatin immunoprecipitation (ChIP). Histones are either methylated or acetylated when demarcating chromatin states. Antibodies against these marks of activation, for example, H3K4me1 or H3K27ac, are therefore used on fixed DNA to precipitate out active histones, and associated DNA. Sequencing of these regions (ChIP-seq) can identify all active regions within different cell types and states. Interaction between disease-associated variants and their target genes, often situated a long distance in a linear view of the chromosome, can be mapped by chromosome conformation technologies, such as $3 \mathrm{C}$, Hi-C and Capture Hi- $\mathrm{C}^{7374}$ (figure 4B). Here the DNA is first fixed within the nucleus in its three-dimensional (3D) conformation. The DNA is then cut, and then reannealed, such that regions that are close together spatially are allowed to join together. Sequencing these reformed regions gives an indication of how close the DNA was in 3D space, such that enhancers are linked to genes through physical interaction. In this way, using both public data and data generated by individual laboratories, it is possible to determine the associated SNPs that are in active regions, the cell types in which this occurs, the gene that is interacting with the region and the effect on expression (figure 4B). For example, GWAS has implicated $\mathrm{T}$ cell involvement via their physical location in two ways: they are proximal to genes important in $\mathrm{T}$ cell immunity, such as HLA, PTPN22, STAT4, TRAF1 and IL2RA; and studies have shown that RA genetic variants are enriched in regions that are open and active in T cells. ${ }^{69}$

Further validation is now being provided by studies employing genetic engineering techniques, specifically CRISPR technology, to directly perturb the DNA regions associated with diseases and measure downstream consequences on cell phenotype. In this way, we are beginning to understand the molecular consequences of carrying a disease risk variant, whether it upregulates or downregulates a gene or pathway, whether this is dependent on cell type or stimulation, and the ultimate consequences on immune function. ${ }^{75}$ 
A

RA genetic risk variants identified by GWAS

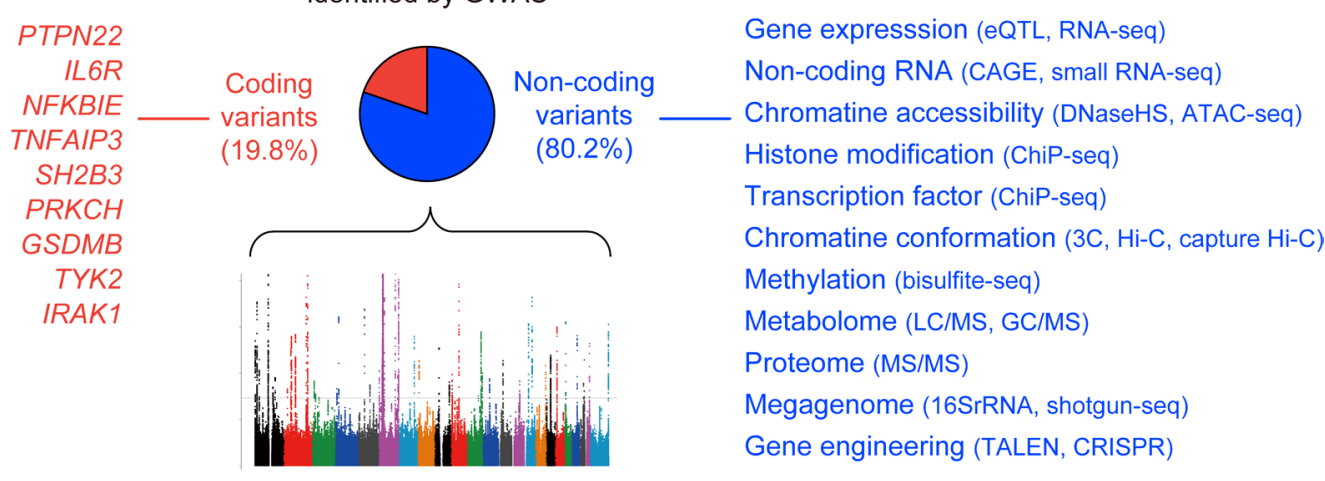

B

(1) GWAS variants

(2) Genomic landscape

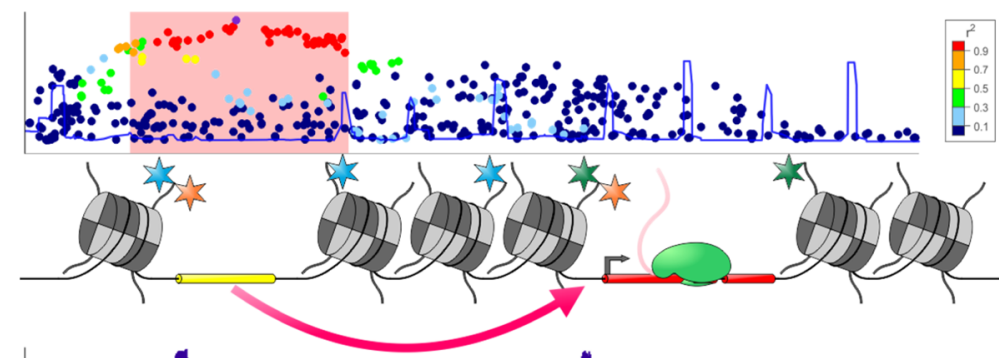

(3) Accessibility

(4) Enhancer boundaries

(5) RNA-seq

(6) $\mathrm{CHi}-\mathrm{C}$

(7) In cell type 2

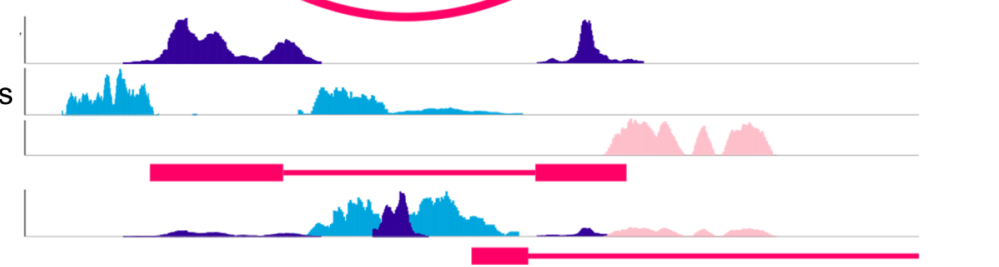

Figure 4 Roles of coding and non-coding risk variants in the genetics of RA. (A) Of the $>100$ RA risk variants, only $20 \%$ are attributable to coding variants such as PTPN22 (R620W) and others. ${ }^{4}$ To understand the roles of the remaining $80 \%$ of the risk variants, we need to integrate RA GWAS results with a variety of omics layer information constructed using the latest technologies (eg, eQTL study involving RNA-seq for exploring gene expression profiles). (B) Demonstrating how omics data can be incorporated into GWAS findings to give an insight into the causal variant and gene. The lead GWAS single nucleotide polymorphism (SNP) (purple dot, row 1) is highly correlated with a number of other SNPs (red dots, row 1) each equally likely to be causal. These SNPs are in an open region of chromatin (yellow, row 2), marked by modified histones (blue, orange and green stars, row 2). The region is open (DNaseHS peaks (purple), row 3), and flanked by modified histones (blue peaks, row 4). This open region (yellow, row 2 ) is interacting ( $\mathrm{CHiC}$, pink bars, row 6) with an expressed gene (pink peaks, row 5). This is not found in a different cell type (row 7). This provides evidence for gene and cell type implicated by the disease GWAS findings. ChiP, chromatin immunoprecipitation; eQTL, expression quantitative trait loci; GWAS, genome-wide association studies; RA, rheumatoid arthritis.

\section{CONCLUSION}

As described above, tremendous progress has been made in understanding the genetics of RA. However, we have yet to fully elucidate the pathogenic mechanism of RA. Genetics-based drug response prediction is currently challenging, in part because the responsible variants and genes are not fully understood. Therefore, we need to move forward applying the various approaches to identify the missing links.

In addition, the problem of missing heritability remains to be solved. GWAS are based on the 'common disease, common variant' hypothesis, which posits that common diseases are attributable to allelic variants present in more than $1 \%-5 \%$ of a population. Rare variants may also contribute to missing heritability, but few examples have been identified thus far. Data from a GWAS of rare variants are difficult to analyse statistically due to the low frequency of occurrence, despite the strong effect. To understand which risk variants are associated with pathogenesis, statistical associations between genetic variation and disease must be linked to functionality and causality. Alternatively, the accumulation of data from ongoing whole-genome sequencing studies could overcome these problems in the near future.

Contributors All authors (YO, SE, AS, YK and KY) wrote a part of the manuscript and $Y O$ and $K Y$ have integrated them. All have reviewed and approved the final manuscript.

Funding This study was funded by Japan Society for the Promotion of Science (grant number 18H05285), Japan Agency for Medical Research and Development (grant number 15545436) and Takeda Pharmaceutical.

Competing interests KY has received honoraria or research grants from AbbVie, Astellas, AYUMI, Bristol-Myers Squibb, Chugai, Eisai, Janssen, Mitsubishi Tanabe, Ono and UCB. YK has received research grants from Takeda Pharm, Chugai Pharm and Pfizer

Patient consent for publication Not required.

Ethics approval RIKEN Ethical Committee.

Provenance and peer review Commissioned; externally peer reviewed.

\section{REFERENCES}

1 Smolen JS, Aletaha D, Barton A, et al. Rheumatoid arthritis. Nat Rev Dis Primers 2018;4:18001. 
2 MacGregor AJ, Snieder H, Rigby AS, et al. Characterizing the quantitative genetic contribution to rheumatoid arthritis using data from twins. Arthritis Rheum 2000;43:30-7.

3 Wellcome Trust Case Control Consortium. Genome-wide association study of 14,000 cases of seven common diseases and 3,000 shared controls. Nature 2007;447:661-78.

4 Okada Y, Wu D, Trynka G, et al. Genetics of rheumatoid arthritis contributes to biology and drug discovery. Nature 2014;506:376-81.

5 GTEx Consortium. Human genomics. The Genotype-Tissue Expression (GTEx) pilot analysis: multitissue gene regulation in humans. Science 2015;348:648-60.

6 Gregersen PK, Silver J, Winchester RJ. The shared epitope hypothesis. an approach to understanding the molecular genetics of susceptibility to rheumatoid arthritis. Arthritis Rheum 1987;30:1205-13.

7 International HapMap Consortium. The International HapMap Project. Nature 2003;426:789-96.

8 Ozaki K, Ohnishi Y, lida A, et al. Functional SNPs in the lymphotoxin-alpha gene that are associated with susceptibility to myocardial infarction. Nat Genet 2002;32:650-4.

9 Suzuki A, Yamada R, Chang X, et al. Functional haplotypes of PADI4, encoding citrullinating enzyme peptidylarginine deiminase 4, are associated with rheumatoid arthritis. Nat Genet 2003;34:395-402.

10 Kochi Y, Yamada R, Suzuki A, et al. A functional variant in FCRL3, encoding Fc receptor-like 3 , is associated with rheumatoid arthritis and several autoimmunities. Nat Genet 2005;37:478-85.

11 Plenge RM, Cotsapas C, Davies L, et al. Two independent alleles at 6q23 associated with risk of rheumatoid arthritis. Nat Genet 2007;39:1477-82.

12 Plenge RM, Seielstad M, Padyukov L, et al. TRAF1-C5 as a risk locus for rheumatoid arthritis--a genomewide study. N Engl J Med 2007;357:1199-209.

13 Remmers EF, Plenge RM, Lee AT, et al. STAT4 and the risk of rheumatoid arthritis and systemic lupus erythematosus. N Engl J Med 2007;357:977-86.

14 Plenge RM, Padyukov L, Remmers EF, et al. Replication of putative candidate-gene associations with rheumatoid arthritis in $>4,000$ samples from North America and Sweden: association of susceptibility with PTPN22, CTLA4, and PADI4. Am J Hum Genet 2005;77:1044-60.

15 Ikari K, Kuwahara M, Nakamura T, et al. Association between PADI4 and rheumatoid arthritis: a replication study. Arthritis Rheum 2005;52:3054-7.

16 Abecasis GR, Auton A, BrooksLD, et al. An integrated map of genetic variation from 1,092 human genomes. Nature 2012;491:56-65.

17 Li Y, Willer C, Sanna S, et al. Genotype imputation. Annu Rev Genomics Hum Genet 2009; 10:387-406.

18 Stahl EA, Raychaudhuri S, Remmers EF, et al. Genome-wide association study meta-analysis identifies seven new rheumatoid arthritis risk loci. Nat Genet 2010;42:508-14.

19 Okada Y, Terao C, Ikari K, et al. Meta-analysis identifies nine new loci associated with rheumatoid arthritis in the Japanese population. Nat Genet 2012;44:511-6.

20 Eyre S, Bowes J, Diogo D, et al. High-density genetic mapping identifies new susceptibility loci for rheumatoid arthritis. Nat Genet 2012;44:1336-40.

21 Kochi Y, Thabet MM, Suzuki A, et al. PADI4 polymorphism predisposes male smokers to rheumatoid arthritis. Ann Rheum Dis 2011;70:512-5.

22 Kurreeman FA, Stahl EA, Okada Y, et al. Use of a multiethnic approach to identify rheumatoid- arthritis-susceptibility loci, 1 p36 and 17q12. Am J Hum Genet 2012;90:524-32.

23 Kim K, Bang SY, Ikari K, et al. Association-heterogeneity mapping identifies an Asian-specific association of the GTF2I locus with rheumatoid arthritis. Sci Rep 2016;6:27563.

24 Okada Y, Muramatsu T, Suita N, et al. Significant impact of miRNA-target gene networks on genetics of human complex traits. Sci Rep 2016;6:22223.

25 Sakaue S, Hirata J, Maeda Y, et al. Integration of genetics and miRNA-target gene network identified disease biology implicated in tissue specificity. Nucleic Acids Res 2018.

26 Zhang Y, Qi G, Park JH, et al. Estimation of complex effect-size distributions using summary-level statistics from genome-wide association studies across 32 complex traits. Nat Genet 2018;50:1318-26.

27 Saxena R, Plenge RM, Bjonnes AC, et al. A multinational arab genome-wide association study identifies new genetic associations for rheumatoid arthritis. Arthritis Rheumatol 2017:69:976-85.

28 Danila MI, Laufer VA, Reynolds RJ. Dense Genotyping of Immune-Related Regions Identifies Loci for Rheumatoid Arthritis Risk and Damage in African Americans. Mol Med 2017;23:1-187

29 Traylor M, Curtis C, Patel H, et al. Genetic and environmental risk factors for rheumatoid arthritis in a UK African ancestry population: the GENRA case-control study. Rheumatology 2017;56:1282-92.

30 López Herráez D, Martínez-Bueno M, Riba L, et al. Rheumatoid arthritis in Latin Americans enriched for Amerindian ancestry is associated with loci in chromosomes 1, 12, and 13, and the HLA class II region. Arthritis Rheum 2013;65:1457-67

31 Pickrell JK, Berisa T, Liu JZ, et al. Detection and interpretation of shared genetic influences on 42 human traits. Nat Genet 2016;48:709-17.
32 Zhernakova A, Stahl EA, Trynka G, et al. Meta-analysis of genome-wide association studies in celiac disease and rheumatoid arthritis identifies fourteen non-HLA shared loci. PLoS Genet 2011;7:e1002004.

33 Chatterjee N, Shi J, García-Closas M. Developing and evaluating polygenic risk prediction models for stratified disease prevention. Nat Rev Genet 2016;17:392-406

34 Stahl EA, Wegmann D, Trynka G, et al. Bayesian inference analyses of the polygenic architecture of rheumatoid arthritis. Nat Genet 2012;44:483-9.

35 Finucane HK, Bulik-Sullivan B, Gusev A, et al. Partitioning heritability by functional annotation using genome-wide association summary statistics. Nat Genet 2015:47:1228-35

36 Lee SH, Byrne EM, Hultman CM, et al. New data and an old puzzle: the negative association between schizophrenia and rheumatoid arthritis. Int J Epidemiol 2015:44:1706-21.

37 Bulik-Sullivan B, Finucane HK, Anttila V, et al. An atlas of genetic correlations across human diseases and traits. Nat Genet 2015;47:1236-41.

38 Oken RJ, Schulzer M. At issue: schizophrenia and rheumatoid arthritis: the negative association revisited. Schizophr Bull 1999;25:625-38.

39 van der Woude D, Houwing-Duistermaat JJ, Toes RE, et al. Quantitative heritability of anti-citrullinated protein antibody-positive and anti-citrullinated protein antibodynegative rheumatoid arthritis. Arthritis Rheum 2009;60:916-23.

40 Kochi Y, Yamada R, Kobayashi K, et al. Analysis of single-nucleotide polymorphisms in Japanese rheumatoid arthritis patients shows additional susceptibility markers besides the classic shared epitope susceptibility sequences. Arthritis Rheum 2004;50:63-71.

41 Padyukov L, Seielstad M, Ong RT, et al. A genome-wide association study suggests contrasting associations in ACPA-positive versus ACPA-negative rheumatoid arthritis. Ann Rheum Dis 2011;70:259-65.

42 The International HIV Controllers Study. The major genetic determinants of HIV-1 control affect HLA class I peptide presentation. Science 2010;330:1551-7.

43 Jia $X$, Han B, Onengut-Gumuscu S, et al. Imputing amino acid polymorphisms in human leukocyte antigens. PLoS One 2013;8:e64683.

44 Raychaudhuri S, Sandor C, Stahl EA, et al. Five amino acids in three HLA proteins explain most of the association between $\mathrm{MHC}$ and seropositive rheumatoid arthritis. Nat Genet 2012;44:291-6.

45 Okada Y, Kim K, Han B, et al. Risk for ACPA-positive rheumatoid arthritis is driven by shared HLA amino acid polymorphisms in Asian and European populations. Hum Mol Genet 2014;23:6916-26.

46 Han B, Diogo D, Eyre S, et al. Fine mapping seronegative and seropositive rheumatoid arthritis to shared and distinct HLA alleles by adjusting for the effects of heterogeneity. Am J Hum Genet 2014;94:522-32.

47 Reynolds RJ, Ahmed AF, Danila MI, et al. HLA-DRB1-associated rheumatoid arthritis risk at multiple levels in African Americans: hierarchical classification systems, amino acid positions, and residues. Arthritis Rheumatol 2014;66:3274-82.

48 Okada Y, Suzuki A, Ikari K, et al. Contribution of a Non-classical HLA Gene, HLA-DOA to the Risk of Rheumatoid Arthritis. Am J Hum Genet 2016;99:366-74.

49 Hirata J, Hosomichi K, Sakaue S. Genetic and phenotypic landscape of the MHC region in the Japanese population. Nat Genet In Press.

50 Bottini N, Musumeci L, Alonso A, et al. A functional variant of lymphoid tyrosine phosphatase is associated with type I diabetes. Nat Genet 2004;36:337-8.

51 Bottini N, Vang T, Cucca F, et al. Role of PTPN22 in type 1 diabetes and other autoimmune diseases. Semin Immunol 2006;18:207-13.

52 Rieck M, Arechiga A, Onengut-Gumuscu S, et al. Genetic variation in PTPN22 corresponds to altered function of T and B lymphocytes. J Immunol 2007:179:4704-10.

53 Vang T, Congia M, Macis MD, et al. Autoimmune-associated lymphoid tyrosine phosphatase is a gain-of-function variant. Nat Genet 2005;37:1317-9.

54 Gregersen PK, Lee HS, Batliwalla F, et al. PTPN22: setting thresholds for autoimmunity. Semin Immunol 2006;18:214-23.

55 Vang T, Landskron J, Viken MK, et al. The autoimmune-predisposing variant of lymphoid tyrosine phosphatase favors T helper 1 responses. Hum Immunol 2013;74:574-85

56 Wang Y, Ewart D, Crabtree JN, et al. PTPN22 Variant R620W Is Associated With Reduced Toll-like Receptor 7-Induced Type I Interferon in Systemic Lupus Erythematosus. Arthritis Rheumatol 2015;67:2403-14.

57 Chang HH, Dwivedi N, Nicholas AP, et al. The W620 polymorphism in PTPN22 disrupts its interaction with peptidylarginine deiminase type 4 and enhances citrullination and NETosis. Arthritis Rheumatol 2015:67:2323-34.

58 Dai X, James RG, Habib T, et al. A disease-associated PTPN22 variant promotes systemic autoimmunity in murine models. J Clin Invest 2013;123:2024-36.

59 Zhang J, Zahir N, Jiang Q, et al. The autoimmune disease-associated PTPN22 variant promotes calpain-mediated Lyp/Pep degradation associated with lymphocyte and dendritic cell hyperresponsiveness. Nat Genet 2011;43:902-7.

60 Hagberg N, Joelsson M, Leonard D, et al. The STAT4 SLE risk allele rs7574865[T] is associated with increased IL-12-induced IFN- $\gamma$ production in T cells from patients with SLE. Ann Rheum Dis 2018:77:1070-7.

61 Kochi Y, Okada Y, Suzuki A, et al. A regulatory variant in CCR6 is associated with rheumatoid arthritis susceptibility. Nat Genet 2010;42:515-9.

62 Ishigaki K, Kochi Y, Suzuki A, et al. Polygenic burdens on cell-specific pathways underlie the risk of rheumatoid arthritis. Nat Genet 2017;49:1120-5. 
63 Honne K, Hallgrímsdóttir I, Wu C, et al. A longitudinal genome-wide association study of anti-tumor necrosis factor response among Japanese patients with rheumatoid arthritis. Arthritis Res Ther 2016;18:12.

64 Umicevic Mirkov M, Cui J, Vermeulen SH, et al. Genome-wide association analysis of anti-TNF drug response in patients with rheumatoid arthritis. Ann Rheum Dis 2013;72:1375-81.

65 Cui J, Stahl EA, Saevarsdottir S, et al. Genome-wide association study and gene expression analysis identifies CD84 as a predictor of response to etanercept therapy in rheumatoid arthritis. PLoS Genet 2013;9:e1003394.

66 Plant D, Bowes J, Potter C, et al. Genome-wide association study of genetic predictors of anti-tumor necrosis factor treatment efficacy in rheumatoid arthritis identifies associations with polymorphisms at seven loci. Arthritis Rheum 2011;63:645-53.

67 Julià A, Fernandez-Nebro A, Blanco F, et al. A genome-wide association study identifies a new locus associated with the response to anti-TNF therapy in rheumatoid arthritis. Pharmacogenomics J 2016;16:147-50.

68 Sieberts SK, Zhu F, García-García J, et al. Crowdsourced assessment of common genetic contribution to predicting anti-TNF treatment response in rheumatoid arthritis. Nat Commun 2016;7:12460.
69 Farh KK, Marson A, Zhu J, et al. Genetic and epigenetic fine mapping of causal autoimmune disease variants. Nature 2015;518:337-43.

70 ENCODE Project Consortium. An integrated encyclopedia of DNA elements in the human genome. Nature 2012;489:57-74.

71 Stunnenberg HG, Hirst M, Abrignani S. The international human epigenome consortium: a blueprint for scientific collaboration and discovery. Cell 2016;167:167-149.

72 Kundaje A, Meuleman W, Ernst J, et al. Integrative analysis of 111 reference human epigenomes. Nature 2015;518:317-30.

73 Schmitt AD, Hu M, Ren B, et al. Genome-wide mapping and analysis of chromosome architecture. Nat Rev Mol Cell Biol 2016;17:743-55.

74 Martin P, McGovern A, Orozco G, et al. Capture Hi-C reveals novel candidate genes and complex long-range interactions with related autoimmune risk loci. Nat Commun 2015;6:10069.

75 Simeonov DR, Gowen BG, Boontanrart M, et al. Discovery of stimulation-responsive immune enhancers with CRISPR activation. Nature 2017;549:111-5. 